\title{
Quantum Thermalization With Couplings
}

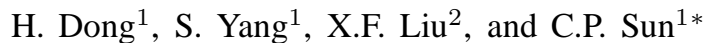 \\ ${ }^{1}$ Institute of Theoretical Physics, Chinese Academy of Science, Beijing 100080, China and \\ ${ }^{2}$ Department of Mathematics, Beijing University, Beijing 100871, China
}

\begin{abstract}
We study the role of the system-bath coupling for the generalized canonical thermalization [S. Popescu, et al., Nature Physics 2,754(2006) and S. Goldstein et al., Phys. Rev. Lett. 96, 050403(2006)] that reduces almost all the pure states of the "universe" [formed by a system $S$ plus its surrounding heat bath $B$ ] to a canonical equilibrium state of $S$. We present an exactly solvable, but universal model for this kinematic thermalization with an explicit consideration about the energy shell deformation due to the interaction between $S$ and $B$. By calculating the state numbers of the "universe" and its subsystems $S$ and $B$ in various deformed energy shells, it is found that, for the overwhelming majority of the "universe" states (they are entangled at least), the diagonal canonical typicality remains robust with respect to finite interactions between $S$ and $B$. Particularly, the kinematic decoherence is utilized here to account for the vanishing of the off-diagonal elements of the reduced density matrix of $S$. It is pointed out that the non-vanishing off-diagonal elements due to the finiteness of bath and the stronger system-bath interaction might offer more novelties of the quantum thermalization.
\end{abstract}

PACS numbers: 05.30.Ch, 03.65.-w, 05.20.Gg

Statistical mechanics is one of the most important and successful areas of modern physics. However its foundation is still debatable and is actually under debate. Most recently a mechanism for universal canonical thermalization has been found in the following meaning: almost all the pure states of the "universe" consisting of the considered system $S$ and its surrounding heat bath $B$ can be reduced into a generalized canonical state by tracing over the bath [1]. Here, the allowed "universe" states lie in a subspace defined by a general constraint $R$, which limits the number of the states to be finite, yet very large. It is noticed that in such generalized thermalization, the constraint $R$ is rather general and need not be the energy shell of the "universe", and generally speaking it doses not lead to the usual canonical state in thermal equilibrium.

When the constraint $R$ is specialized as a total energy shell, the generalized canonical state becomes a usual canonical thermal state. Such a thermalization was described by the canonical typicality in ref. [2] associated with the so called overwhelming majority rule, based on the law of large numbers, in counting the state numbers of the constrained "universe" and its subsystems. Significant results concerning such canonical typicality have been obtained by several authors for different purposes [3, 4, 5, 6, 7, 8]. Actually, to derive the canonical distribution of $S$ from the microcanonical density matrix [9] or an entangled pure state [2] of the "universe" $U=S+B$, the interaction between $S$ and $B$ should be weak enough to allow a physical partition for the "universe".

In this paper, we will quantitatively consider the effect of system-bath coupling on the above mentioned kinematics of canonical thermalization leading to equilibrium canonical state. The present investigation only concerns the usual canonical state. In the weak interaction limit, the inverse temperature $\beta=\partial S(E) / \partial E$ emerges from the thermodynamic entropy $S(E)=\ln \Omega(E, \delta)$ where $\Omega(E, \delta)$ is the microstate number of the bath in the energy shell $[E, E+\delta]$. Since the interaction between $S$ and $B$ deforms the geometry of the energy shell, we will reexamine the validity of the temperature definition from the new perspective offered by the generalized thermalization [1, 2]. We try to understand how the offdiagonal elements of the reduced density matrix of $S$ vanish due to the factorization structure, in association with the random phase explanation [10, 11]. We find that if the mode number of the bath is not large enough and the system-bath coupling is strong enough, generally there exist nonzero offdiagonal elements in the reduced density matrix. Notice that these nonzero off-diagonal elements introduce quantum coherence into the usual thermal equilibrium state and thus result in novel thermodynamic features.

We begin with a universal model: the system $S$ we consider is an $M$-level system with the Hamiltonian $H_{S}=$ $\sum \epsilon_{n}|n\rangle\langle n|$, where $|n\rangle$ is the eigenstate with eigenvalue $\epsilon_{n}$, $n=1,2, . ., M$; and the bath $B$ is modeled as a collection of $N$ harmonic oscillators of frequencies $\omega_{j}(j=1,2, . . N)$ with the Hamiltonian $H_{B}=\sum_{j} \omega_{j} a_{j}^{\dagger} a_{j}$. This model can be regarded as a universal approach, because in the weak coupling limit, any heat bath could be universally modeled as a collection of harmonic oscillators with the linear couplings to the surrounded system according to the proofs in ref. [13]. For this reason, the interaction $H_{I}$ between $B$ and $S$ should be modeled to be linear with respect to the bath variables $a_{j}^{\dagger}$ and $a_{j}$. So we assume a simplest system-bath coupling

$$
H_{I}=\sum_{j, n} \lambda_{n}|n\rangle\langle n|\left(g_{j} a_{j}^{\dagger}+\text { H.c. }\right)
$$

where $\lambda_{n}$ are real numbers. Note that $H_{I}$ is of non-demolition since $\left[H_{S}, H_{I}\right]=0$. Thus the interaction only causes the dephasing of $S$, and the energy dissipation of $S$ will not appear [11, 12].

Obviously, the eigenvalues of the "universe" formed by $B$ and $S$ are

$$
E\left(n,\left\{n_{j}\right\}\right)=\epsilon_{n}(\kappa)+\sum_{j=1}^{N} n_{j} \omega_{j}
$$




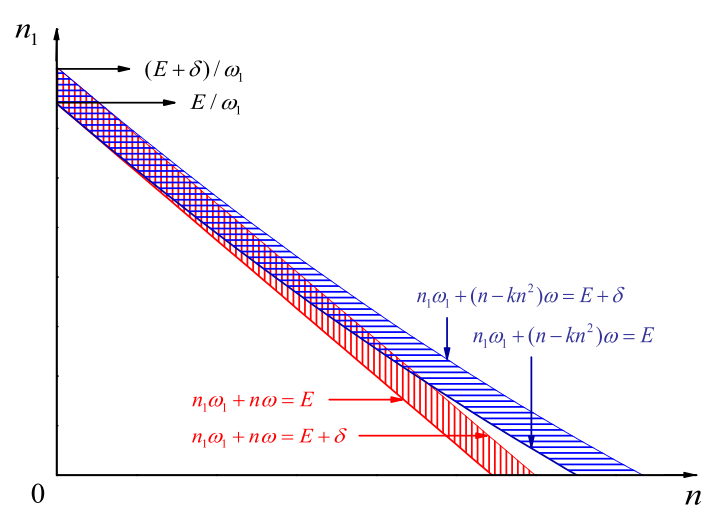

FIG. 1: (Color Online) Geometrical deformation of the energy shell. Here we show explicitly the deformation, when $S$ is a harmonic oscillator. (a) Without the interaction, the energy shell is the red area; (b) The interaction deforms the red area into the blue area.

corresponding to the eigenstates $\left|n,\left\{n_{j}\right\}\right\rangle=|n\rangle \otimes$ $\prod_{j=1}^{N}\left|n_{j}(n)\right\rangle$, where $\left|n_{j}(n)\right\rangle=D\left(\alpha_{j n}\right)\left|n_{j}\right\rangle$ is defined in terms of the Fock states $\left|n_{j}\right\rangle$ of $B$ and the coherent-stategenerating operator $D\left(\alpha_{j n}\right)=\exp \left(\alpha_{j n} a_{j}^{\dagger}-h . c\right)$ with the displacement parameters $\alpha_{j n}=-\lambda_{n} g_{j} /\left(2 \omega_{j}\right) ; \epsilon_{n}(\kappa)=$ $\epsilon_{n}-\kappa \lambda_{n}^{2}$. Especially, the parameter $\kappa=\Sigma_{j}\left|g_{j}\right|^{2} /\left(4 \omega_{j}\right)$ reflects the role of the interaction between $S$ and $B$.

Note that the system-bath coupling deforms the energy shell of thickness $\delta$ defined by the total constraint $E \leq$ $E\left(n,\left\{n_{j}\right\}\right) \leq E+\delta$. This energy shell determines a subset of the "universe" states. For convenience, we denote by $V(E, \delta)$ the subspace spanned by this subset. To have a clear picture of this deformation of energy shell, let us consider the following simple example: the system $S$ is a harmonic oscillator with frequency $\omega$ and $\lambda_{n}=n$. In this case, the renormalized energy of $S$ is $E_{s}(n)=n \omega-\kappa n^{2}$. In Fig. 1, we illustrate the deformation of the energy shell when $N=1$. If there were no interaction, the above constraint would produce the red area. The interaction introduces the nonlinear term $\kappa n^{2}$ to deform it into the blue area.

Generally, in order to derive the canonical distribution of $S$ from an entangled pure state of the "universe", we need to calculate the dimension $\Omega_{N+1}(E, \delta, \kappa)$ of $V(E, \delta)$ and the dimension $\Omega_{N}\left(E-\epsilon_{n}, \delta, \kappa\right)$ of the subspace $V^{B}(n, \kappa)$ spanned by the states with the constraint

$$
E-\epsilon_{n}(\kappa) \leq \sum_{j} n_{j} \omega_{j} \leq E+\delta-\epsilon_{n}(\kappa)
$$

Let us start with a pure entanglement state $|\psi\rangle=$ $\sum^{\prime} C\left(n,\left\{n_{j}\right\}\right)\left|n,\left\{n_{j}\right\}\right\rangle$ of the constrained "universe", where $\sum^{\prime}$ denotes the summation under the total constraint. By tracing over the variables of $B$, the reduced density matrix
$\rho_{S}=\operatorname{Tr}_{B}(|\psi\rangle\langle\psi|)$ of $S$ is obtained as

$$
\rho_{S}=\sum_{n} P_{n}|n\rangle\left\langle n\left|+\sum_{n \neq m} F_{n m}\right| n\right\rangle\langle m|,
$$

where the diagonal elements are $P_{n}=P_{n}(E, \kappa)=$ $\sum^{\prime \prime}\left|C\left(n, n_{j}\right)\right|^{2}$ and $\sum^{\prime \prime}$ stands for the summation under the constraint (3). We represent this summation by the summation over the constrained indices $\left[n_{j}\right]_{E, n}$, then the off-diagonal elements can be written as

$$
F_{n m}=\sum_{\left[m_{j}\right]_{E, m}} \sum_{\left[n_{j}\right]_{E, n}} C\left(n, n_{j}\right) C^{*}\left(m, m_{j}\right) D_{m\left(m_{j}\right)}^{n\left(n_{j}\right)} .
$$

where

$$
D_{m\left(m_{j}\right)}^{n\left(n_{j}\right)}=\prod_{j=1}^{N} d_{m_{j}(m)}^{n_{j}(n)}=\prod_{j=1}^{N}\left\langle m_{j}(m) \mid n_{j}(n)\right\rangle
$$

are decoherence factors with a factorized structure and for $m \neq n, m_{j} \geq n_{j}$, each factor

$$
d_{m_{j}(m)}^{n_{j}(n)}=\Delta_{\alpha}^{\left(m_{j}-n_{j}\right)} e^{-\Delta_{\alpha}^{2} / 2} L_{n_{j}}^{\left(m_{j}-n_{j}\right)}\left(\Delta_{\alpha}^{2}\right) \sqrt{\frac{n_{j} !}{m_{j} !}}
$$

is expressed in terms of the associated Laguerre polynomials $L_{n}^{m}(x)$ with the variable $\Delta_{\alpha}=$ $-g_{j} /\left(2 \omega_{j}\right)(n-m)$.

In order to show the generalized quantum thermalization, we need to study how the diagonal elements of $\rho_{S}$ approach the Gibbs distribution $P_{n} \propto p_{n}^{G}=\exp \left(-\beta \epsilon_{n}\right)$, while the off-diagonal elements $F_{n m}$ vanish as a quantum decoherence effect. To this end, we use the basic assumptions for statistical mechanics that $\delta \ll \omega$, but $\delta \gg \omega_{j}$ and $N \rightarrow \infty$.

First, we consider the diagonal elements. Let us establish the formula

$$
P_{n}(E, \kappa)=\frac{\Omega_{N}\left(E-\epsilon_{n}, \delta, \kappa\right)}{\Omega_{N+1}(E, \delta, \kappa)} .
$$

in some sense under the reasonable assumption that $\left|C\left(n,\left\{n_{j}\right\}\right)\right|^{2}$ are random variables with an identical distribution. In fact, if this condition is satisfied, then these random variables have the same mathematical expectation $\left(\Omega_{N+1}(E, \delta, \kappa)\right)^{-1}$ due to the restriction $1=\sum^{\prime}\left|C\left(n,\left\{n_{j}\right\}\right)\right|^{2}$. It then follows from the law of large numbers that $\sum^{\prime \prime}\left|C\left(n,\left\{n_{j}\right\}\right)\right|^{2}$ approaches $\Omega_{N}\left(E-\epsilon_{n}, \delta, \kappa\right)\left(\Omega_{N+1}(E, \delta, \kappa)\right)^{-1}$ in probability. Namely, the above formula is valid with high probability. Here we remark that with a straightforward calculation (e.g., in Ref. [2]), this formula can be obtained from the microcanonical state $\rho_{M C}=$ $\sum^{\prime}\left[1 / \Omega_{N+1}(E, \delta, \kappa)\right]\left|n,\left\{n_{j}\right\}\right\rangle\left\langle n,\left\{n_{j}\right\}\right|$ of the "universe".

We are now in a position to consider how $P_{n}(E, \kappa)$ leads to the canonical distribution. According to Refs. [1, 2], when there is no interaction, $P_{n}(E, \kappa=0)$ does give rise to the canonical distribution for almost all the pure states $|\psi\rangle$ of the 
constrained "universe". But when there exists an interaction between $S$ and $B$, is it still the case? The answer seems to be positive when the interaction is weak. We will attack this problem by theoretical analysis and numerical simulation.

If we can show that $P_{n}(E, \kappa)$ possesses a "conformal invariance" with respect to the geometrical deformation of the energy shell caused by the interaction, i.e., $P_{n}(E, \kappa) \sim$ $P_{n}(E, \kappa=0)$, then the problem is solved. We try to justify this "conformal invariance" for weak interaction $(\kappa \neq 0)$. It follows from the direct sum decomposition $V(E, \delta)=$ $\sum \oplus V^{B}(n, \kappa)$ of the Hilbert space $V(E, \delta)$ that the dimension of $V(E, \delta)$ can be written as $\Omega_{N+1}(E, \delta, \kappa)=$ $\sum_{n=1}^{M} \Omega_{N}(n)$. Here, $\Omega_{N}(n)$ stands for the number of states in the area defined by Eq. (3), and $M$ is an upper bound of the summation range, which is determined by the positiveness of both the bath energy and the system energy. By straightforward calculation we obtain

$$
\Omega_{N}(n) \simeq \frac{\left(E-\epsilon_{n}(\kappa)\right)^{N-1} \delta}{(N-1) ! \prod_{j=1}^{N} \omega_{j}^{2}}
$$

for very small $\delta$. Thus the diagonal elements of $\rho_{S}$ take the form

$$
P_{n}=\frac{\left[E-\epsilon_{n}(\kappa)\right]^{N-1}}{\sum_{n=1}^{M}\left[E-\epsilon_{n}(\kappa)\right]^{N-1}} .
$$

with a finite $M$ and a large $N$. Since the eigen-energy $\epsilon_{n}$ of the system is much smaller than the total energy of the energy shell, we have $\left[E-\epsilon_{n}(\kappa)\right]^{N-1} \approx \exp [(N-1) \ln (E+$ $\left.\left.\kappa \lambda_{n}^{2}\right)\right] \exp \left(-\beta_{n} \epsilon_{n}\right)$ where $\beta_{n}$ is the quasi-temperature defined as

$$
\beta_{n}=\left.\frac{\partial S(E)}{\partial E}\right|_{E \rightarrow E+\kappa \lambda_{n}^{2}}=\frac{N-1}{E+\kappa \lambda_{n}^{2}} .
$$

Usually the energy correction $\kappa \lambda_{n}^{2}=\lambda_{n}^{2} \Sigma_{j}\left|g_{j}\right|^{2} /\left(4 \omega_{j}\right)$ is much smaller than the shell energy $E$, thus the quasitemperature becomes independent of $n: \beta_{n} \approx N / E$. Therefore, $P_{n}$ is "conformably invariant" with respect to $\kappa$, and for this reason we can expect the Gibbs distribution $P_{n}=$ $\exp \left(-\beta_{n} \epsilon_{n}\right) /\left[\sum_{n=1}^{M} \exp \left(-\beta_{n} \epsilon_{n}\right)\right]$.

It should be pointed out that the above argument is only heuristic since neither the sum $\sum \Omega_{N}(n)$ nor the distribution $P_{n}$ is calculated analytically. Thus we will resort to numerical simulation to support our expectation. Let us assume the system is a harmonic oscillator. In this case, $\lambda_{n}=n$ and the quasi-temperature of the system is exactly $\beta_{n}=$ $(N-1) /\left(E-\kappa n^{2}\right)$. Due to the weakness of interaction, $\beta_{n}$ is approximately equal to $N / E$ when $n$ is small. We choose $E=0.5$ and $\omega=10^{-3}$ and display in Fig 2 the relationship between the distribution $P_{n}$ and $n$ for different $\kappa$ when $N=50$. It is clearly shown that $P_{n}$ indeed decays exponentially as Gibbs distribution as $n$ increases. Tab. I gives the inverse temperature of the system for different coupling parameters $\kappa$. Here, the theoretical inverse temperature is $\beta=98$.

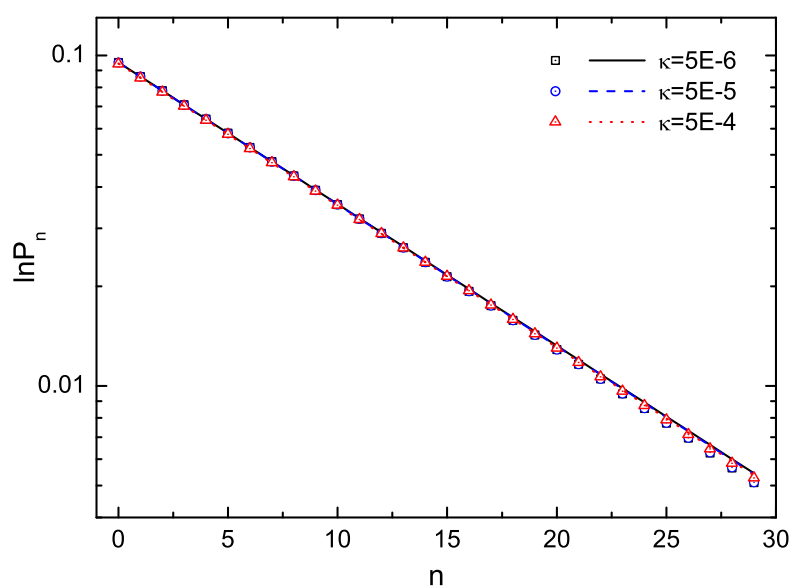

FIG. 2: (Color Online) Plot of $\ln _{n}$ as a function of the number $n$ of the system, when $N=50$ with different $\kappa: 5 \times 10^{-6}(\square)$, $5 \times 10^{-5}(\bigcirc)$ and $5 \times 10^{-4}(\triangle)$. The fitting of the Gibbs distribution is shown by the corresponding lines.

These numerical results demonstrate that the state obtained by tracing over the bath is just the Gibbs canonical state under some conditions.

TABLE I: Inverse Temperature vs Interaction

\begin{tabular}{llll}
\hline$\kappa$ & $5 \times 10^{-6}$ & $5 \times 10^{-5}$ & $5 \times 10^{-4}$ \\
\hline$\beta$ & 98.94 & 98.85 & 98.69 \\
\hline
\end{tabular}

Now we consider the bath-induced decoherence effect, which causes the off-diagonal elements of $\rho_{S}$ to approach zero. When the system-bath couplings can be ignored in comparison with the energy level spacing and the total energy of the bath, any two subspaces defined by the constraint (3) can not overlap each other and then the decoherence factor vanishes. In present situation, the system-bath coupling will weaken this decoherence for thermalization since the positive terms $\lambda_{n+1}^{2}-\lambda_{n-1}^{2}$ reduce the effective system's energy spacings

$$
\epsilon_{n+1}(\kappa)-\epsilon_{n}(\kappa)=\epsilon_{n+1}-\epsilon_{n}-\kappa\left(\lambda_{n+1}^{2}-\lambda_{n-1}^{2}\right)
$$

to make them comparable to the thickness $\delta$ of the energy shell. Then two subspaces $V^{B}(n, \kappa)$ and $V^{B}(m, \kappa)$ of $B$ can overlap each other and the off-diagonal elements $F_{n m}$ will not vanish. However, notice that the norm of each component $d_{m_{j}(m)}^{n_{j}(n)}$ in the decoherence factor is less than unity. Thus the decoherence factor $D$ may still vanish in the thermodynamic limit $N \rightarrow \infty$. So we can say that the factorized structure of $D$ enhances the decoherence [10, 11], and to some extent compensates the negative effect of interaction in thermalization.

Next, we wish to point out that in the mesoscopic case, that is to say, $N$ is not large enough, novel effects may arise. In this 
case, if the system-bath coupling is strong, there will exist finite off-diagonal elements $F_{n m}$ in the reduced density matrix. This means quantum coherence is introduced into the usual thermally equilibrium state. Such a state is called a quasithermal state.

For a two level system with single energy spacing $\Delta$, the quasi-thermal state can be described by the reduced density matrix

$$
\rho_{S}=\left[\begin{array}{cc}
p_{+} & F \\
F^{*} & p_{-}
\end{array}\right]
$$

The diagonal elements $p_{ \pm}=1 /(1+\exp ( \pm \beta \Delta))$ approach the standard Gibbs distributions while the off-diagonal elements are non-vanishing: $F=F_{12}$. We can diagonalize the above reduced density matrix to obtain the two effective probabilities $P_{ \pm}(F)$ exactly. For small $F, P_{ \pm}(F)$ can be approximated as

$$
P_{ \pm}(F) \approx p_{ \pm} \mp \operatorname{coth}\left(\frac{\beta \Delta}{2}\right)|F|^{2}
$$

Then the von Neumann entropy $S_{V N}=$ $-\sum_{\alpha= \pm} P_{\alpha}(F) \ln P_{\alpha}(F)$ is approximated as

$$
S_{V N} \simeq S(E)-\beta \Delta|F|^{2} \operatorname{coth}\left(\frac{\beta \Delta}{2}\right)
$$

It is observed that due to the system-bath interaction the von Neumann entropy explicitly deviates from the thermodynamic entropy

$$
S(E) \simeq \frac{\beta \Delta}{e^{\beta \Delta}+1}+\ln \left(e^{-\beta \Delta}+1\right),
$$

which is by definition the entropy of the Gibbs equilibrium state $\rho_{G}=\operatorname{diag}\left(p_{+}, p_{-}\right)$. Therefore, generally von Neumann entropy does not relate to the meaningful usual notion of temperature. In fact, there exists no good physical notion of temperature for a general non-thermal-equilibrium. But the erasure of quantum information by thermalization indicates where temperature enters in this matter.

However, only for two-level system or system with homogeneous energy level spacing can we define an effec-

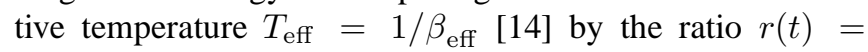
$P_{+}(F) / P_{-}(F)=\exp \left(-\beta_{\text {eff }} \Delta\right)$ and the level spacing $\Delta$. For such a system interacting with a finite heat bath, even in a non equilibrium state, we can imagine that it is in a virtual equilibrium state with the effective inverse temperature

$$
\beta_{\mathrm{eff}} \simeq \beta+\frac{4|F|^{2}}{\Delta} \cosh ^{2}\left(\frac{\beta \Delta}{2}\right) \operatorname{coth}\left(\frac{\beta \Delta}{2}\right)
$$

This effective temperature in the quasi-thermal state is higher than the usual equilibrium temperature. Such kind of quasithermal state with a bit of quantum coherence can demonstrate various exotic natures in thermodynamical processes. Scully et al [15] have proposed a quantum Carnot engine in which the bath atoms are given some quantum coherence, which can increase the effective temperature of the radiation field. In this case, though the second law of thermodynamics is not violated, the quantum Carnot engine possesses some features that are not possible in a classical case.

In summary, the quantum kinetic thermalization of the system is explored, based on an exactly solved and general model with weak interaction between $S$ and $B$ and the effect of interaction is demonstrated as the deformation of the energy shell; Based on the model, we realized the thermalization by tracing over the variable of the bath $B$. Here decoherence is used to account for the disappearance of the off-diagonal elements of the reduced density matrix of the system in contact with a bath of infinitely large particle number in the weak coupling limit. Moreover, the novel thermodynamic effects are emphasized that can result from the non-vanishing off-diagonal elements of the reduced density matrix when the bath is mesoscopic.

This work is supported by the NSFC with grant Nos. 90203018, 10474104 and 60433050, and NFRPC with Nos. 2006 CB921206 and 2005CB724508.

* Electronic address: suncp@itp.ac.cn URL: www.itp.ac.cn/ suncp

[1] S. Popescu, A. J. Short and A. Winter, Nature Physics 2, 754 758 (2006)

[2] S. Goldstein, J. L. Lebowitz, R. Tumulka and N. Zanghì, Phys. Rev. Lett. 96, 050403 (2006)

[3] J. Gemmer and M. Michel, Europhys. Lett. 73, 1-7 (2006).

[4] P. Bocchieri, and A. Loinger, Phys. Rev. 114, 948-951 (1959).

[5] S. Lloyd, Ph.D Thesis, Rockefeller Univ. (1988).

[6] H. Tasaki, Phys. Rev. Lett. 80, 1373-1376 (1998).

[7] M. Michel, G. Mahler, and J. Gemmer, Phys. Rev. Lett. 95, 180602 (2005).

[8] H. P. Breuer, J. Gemmer and M. Michel, Phys. Rev. E 73, 016139 (2006).

[9] L. D. Landau and E. M. Lifshitz, Statistical Physics (Pergamon, London, 1958).

[10] C.P. Sun, Phys. Rev. A48, 898 (1993); C.P. Sun, X.X. Yi and X.J. Liu, Fortschr. Phys. 43, 585 (1995).

[11] P. Zhang, X.F. Liu and C.P. Sun, Phys. Rev. A 66, 042104 (2002).

[12] Y.B. Gao and C.P. Sun, Phys. Rev. E 75 , 011105 (2007).

[13] A. O. Caldiera and A. J. Leggett, Ann. Phys. (NY) 149, 374(1983).

[14] H.T. Quan, Yu-xi Liu, C.P. Sun and Franco Nori, quant-ph/0611275. (2006).

[15] M. O. Scully, M. S. 1 Zubairy, G. S. Agarwal and H. Walther, Science 299, 862, (2003) 